\title{
Non-isothermal volume change behavior of saturated sand subjected to minimal vertical effective stress
}

\author{
Yize $\operatorname{Pan}^{1}$ and Alessandro F. Rotta Loria ${ }^{1, *}$ \\ ${ }^{1}$ Northwestern University, Department of Civil and Environmental Engineering, Mechanics and Energy Laboratory, Evanston, 60208, \\ United States of America
}

\begin{abstract}
To date, the majority of the available studies on the volume change behavior of soils under nonisothermal conditions have focused on fine-grained soils. Only a limited number of investigations have been made available about coarse-grained soils despite their abundance in practice, with contradictory features. To enrich the current knowledge about the volume change behavior of coarse-grained soils under non-isothermal conditions, this investigation presents the results of oedometric tests with temperature control performed on saturated sand. The influence of relative density on the volume change behavior of the tested material under non-isothermal conditions is investigated under a minimal level of applied vertical effective stress. The results show an expansive volume change upon heating and contractive volume change upon cooling for all relative densities. The magnitude of the contraction is more significant than that of the expansion, leading to a residual contractive volume change after one cycle of heating and cooling. The results of this study enrich the current literature about the volume change behavior of coarse-grained soils under non-isothermal conditions. Such competence may be considered for applications at the interface of geomechanics and energy wherein temperature variations occur and characterize the response of coarse-grained soils.
\end{abstract}

\section{Introduction}

Geomaterials, such as coarse-grained soils and finegrained soils, are continuously subjected to temperature variations due to natural or anthropogenic perturbations that involve the transfer of heat. These temperature variations inherently induce deformations of the constituents of such materials at the microscopic scale, which can result in volume changes at the macroscopic scale. The considered deformations influence the equilibrium of the subsurface and can affect the performance of a variety of technologies embedded therein, such as energy geotechnologies [1]. By far, the majority of the available studies on the volume change behavior of soils under non-isothermal conditions have focused on fine-grained soils. Only a limited number of investigations are available about coarse-grained soils despite their abundance in practice, with contradictory features related to their volume change behavior under non-isothermal conditions [2].

The volume change behavior of coarse-grained soils under non-isothermal conditions can be investigated upon and after the thermal loading/unloading process. Information on these aspects instructs about the deformation of the considered materials under nonisothermal conditions. Loading of coarse-grained soils induced by temperature variations is typically associated with heating while unloading with cooling.

To date, some experimental and numerical investigations have addressed the volume change behavior of coarse-grained soils under non-isothermal conditions. The available experimental investigations have resorted to oedometric and triaxial apparatuses with temperature control [3-7]. The available numerical investigation has resorted to finite element simulations [8]. In this context, expansive and/or contractive volume changes upon heating have been shown to characterize coarse-grained soils at the macroscopic scale, despite the individual grain constituting such materials only expand upon heating at the microscopic scale. Contractive volume changes have been observed upon cooling. The contractive volume changes upon heating have been indicated to increase for higher values of applied stress, despite highlighting conflicting features in different investigations [6,7], and to be markedly characterized by the relative density of the material before the loading process [3]. In particular, an interacting role of stress level and relative density seems to govern the non-isothermal volume change behavior of coarse-grained soils. At high stress levels (order of MPa), contractive volume changes have been reported upon both heating and cooling for coarse-grained soils with low to high relative densities [4]. At moderate stress levels (order of $\mathrm{kPa}$ ), initially contractive and subsequently expansive volume changes have been reported upon heating for coarse-grained soils with low relative densities [3,6,7], while purely expansive volume changes upon heating have been found for coarse-grained soils with higher relative densities (that are always associated with contractive volume changes upon cooling) $[3,5]$.

\footnotetext{
* Corresponding author: af-rottaloria@northwestern.edu
} 
This evidence suggests that the influence of relative density on the volume change of coarse-grained soils caused by heating may be hindered by the increase of the stress level, resulting in purely expansive strains for stress levels that tend to zero [2].

Until recently, no information about the nonisothermal volume change of coarse-grained soils with different relative densities before the application of thermal cycles under minimal applied vertical effective stress had been reported. This fact has made the studies on the volume change of coarse-grained soils upon the application of heating and cooling cycles incomplete. In this context, Pan et al. [9] have carried out an experimental and numerical investigation to analyze the volume change behavior of coarse-grained soils under non-isothermal conditions for minimal values of vertical effective stress. The present paper expands on the experimental tests and results of the referenced study, aiming to advance the understanding of the volume change behavior of coarse-grained soils under nonisothermal conditions for low values of applied vertical effective stress.

In the following, the features of the experimental testing campaign are discussed first. Then, the obtained experimental results are presented and discussed. Finally, a summary of the key conclusions that can be drawn from this work is reported. The adopted sign convention in this paper considers compressive stresses, expansive strains and settlements as positive.

\section{Methodology}

The features of the experimental testing campaign are illustrated in this section. The experimental setup and the properties of the investigated material are presented first. Finally, the details of the oedometric tests are illustrated.

\subsection{Experimental setup}

Oedometer apparatuses adapted to control temperature were employed to experimentally investigate the volume change behavior of coarse-grained soils under temperature variations (Fig. 1). In the considered experimental facility, temperature variations are applied by the circulation of water pumped through a spiral tube positioned around the specimens and connected to a heater. The heater includes a thermostat that allows imposing the temperature of the fluid circulating in the spiral tube on a connected bath. The oedometer cells are insulated from heat losses with a polystyrene box. Two tests in series can run simultaneously on two oedometers using one temperature control system. The temperature in the oedometer cell is measured using thermocouples (type-K, accuracy of $0.1^{\circ} \mathrm{C}$ ). The vertical displacement of the specimen is measured by a Linear Variable Differential Transformer (LVDT) placed atop the cap. The oedometer ring embedding the specimen is made of invar to limit the effect of the container radial expansion [3] (an alloy FeNi36, linear coefficient of thermal expansion of $1.2 \times 10^{-6} 1 / \mathrm{K}$ ) and consequently involve negligible boundary effects [8]. Comprehensive technical details of the apparatus have been previously reported by Di Donna and Laloui [10].

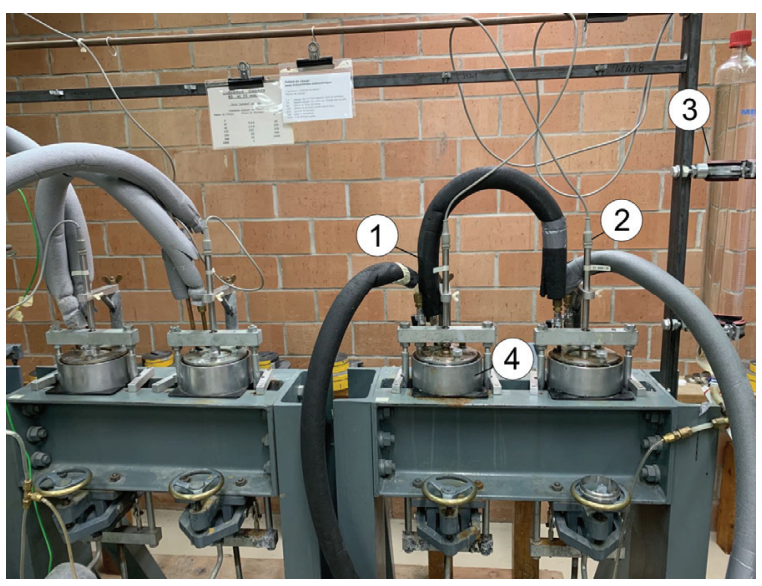

Fig. 1. Oedometer apparatuses with a temperature control system (1: tubes circulating water for temperature control; 2 : LVDT; 3: water supplier; 4: oedometer cell)

\subsection{Investigated material}

The investigated material is reconstituted Foundry sand, which is saturated with de-aired and distilled water. The specific gravity of the material is 2.67 , the maximum and minimum void ratios are 0.80 and 0.52 , respectively. Fig. 2 shows the particle size distribution of Foundry sand ( 0.1 $\mathrm{mm}-0.4 \mathrm{~mm}$ ), as well as its relatively rounded particles from a microscope image. The particle size distribution provides a uniformity coefficient of 1.6. Material specimens are prepared by the air-pluviation method [11], uniformly depositing dry sand into the cylindrical mold of diameter $D=60 \mathrm{~mm}$ and height $H=15 \mathrm{~mm}$.

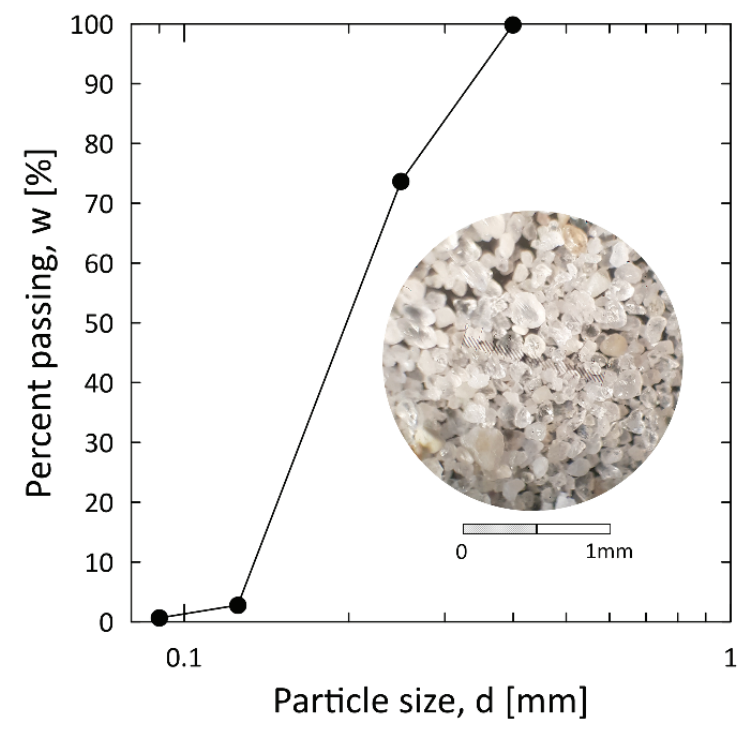

Fig. 2. Particle size distribution and particle shape of Foundry sand 
Three different relative densities of $30 \%, 60 \%$ and $90 \%$, associated with loose, medium dense, and very dense states [12], are prepared [13,14]. To this aim, the mass of material deposited in the cylindrical mold is controlled. Following deposition and placement of a top cap (associated with the imposition of a minimal mechanical load to the specimen), the material is slowly saturated with an upward flow of water to avoid particle movements.

\subsection{Isothermal oedometric tests}

Standard mechanical oedometric tests under isothermal conditions represented by an approximately constant room temperature of $20^{\circ} \mathrm{C}$ were conducted on Foundry sand to provide a mechanical characterization of the Foundry sand. The tests were conducted on saturated specimens with initial relative densities of $10 \%$ and $20 \%$, as a broader experimental campaign was considered to address the (non-isothermal) volume change behavior of material specimens under various stress levels. The average of two parallel results conducted for each relative density is shown in Fig. 3.

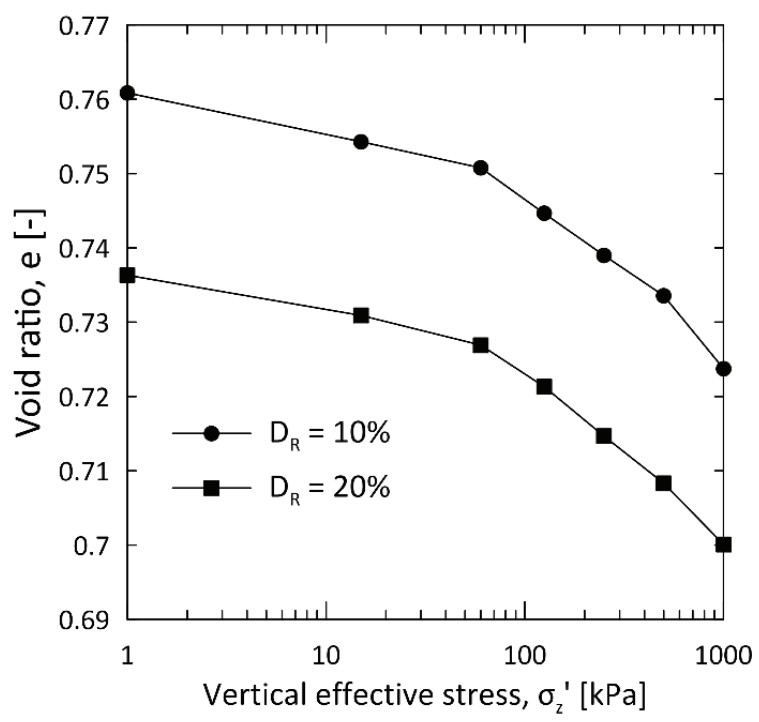

Fig. 3. Volume change behavior of Foundry sand at relative densities of $10 \%$ and $20 \%$

\subsection{Oedometric tests with temperature control}

Extended thermo-mechanical oedometric tests under nonisothermal conditions were conducted on Foundry sand. Specimens with three relative densities of $30 \%, 60 \%$ and $90 \%$ were tested by applying one drained heating-cooling cycle in the temperature range of $20^{\circ} \mathrm{C}-60{ }^{\circ} \mathrm{C}-20^{\circ} \mathrm{C}$ and with a step of $10{ }^{\circ} \mathrm{C}$ considered for both heating and cooling. Fig. 4 shows the representative thermal paths imposed in the previous tests. In all of the tests presented in the following, the vertical effective stress was kept constant at $1 \mathrm{kPa}$, with the oedometer cap placed atop the specimens. No additional mechanical load was applied to specimens. The average of two parallel test results for each relative density was used for analysis.

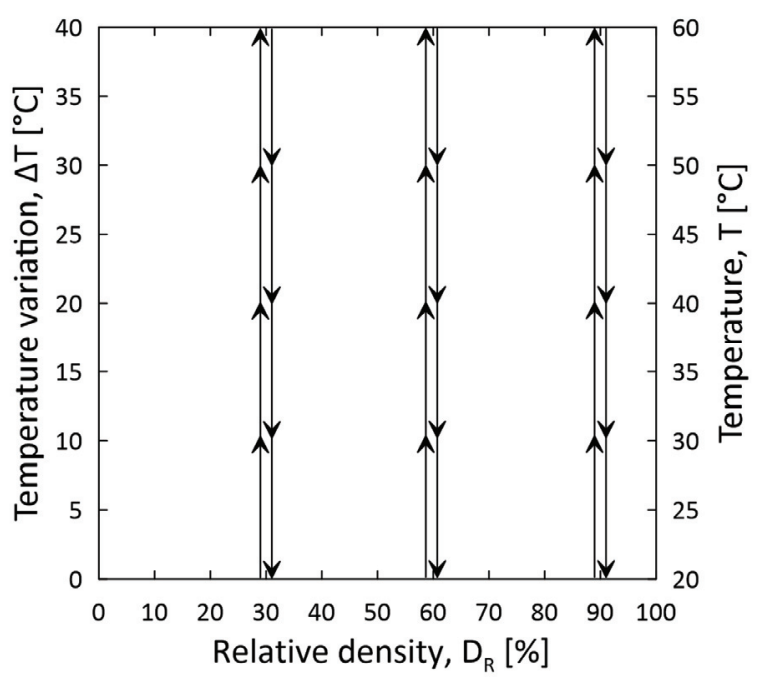

Fig. 4. Loading paths considered in the oedometric tests with temperature control. The paths show the temperature variations of one heating-cooling cycle with a range of $40^{\circ} \mathrm{C}$ and a step of $10^{\circ} \mathrm{C}$

\subsection{Calibration tests}

Two series of calibration tests were conducted to ensure the precision and reliability of the experimental results. First, considering the heat transfer and losses within the oedometer cells, calibration tests were conducted to determine the applied temperature in the heater for each loading or unloading step, and the required waiting time to reach a steady and uniform temperature field within the tested specimen. The temperature inside a trial sand specimen was monitored by placing an additional thermocouple in the middle of the specimen. Fig. 5 shows the variations of the temperature measured in the specimen and the applied temperature in the heater for each thermal loading or unloading step. The results indicate that one hour and a half is necessary to reach a steady temperature field. This result is associated with the noteworthy thermal losses characterizing this system.

Then, calibration tests measuring the displacements induced by the thermal deformation of the apparatuses were performed to apply necessary corrections for the analysis of the results. A dummy specimen made of steel of known physical properties was used to characterize the thermal deformation of the apparatuses [15]. The calibration tests followed the same temperature range and step as the planned oedometric tests with temperature control. Fig. 6 shows the apparatus displacements induced by the temperature variations for the two oedometers. The displacements caused by the thermal deformation of the apparatus $\Delta h_{a}$ were subtracted from the displacements measured in real tests $\Delta h_{r}$ to get the actual displacements of material $\Delta h_{m}$ at sought temperatures:

$$
\Delta h_{m}=\Delta h_{r}-\Delta h_{a}
$$


Knowledge of the actual displacement of the specimen allowed to calculate for each thermal loading or unloading step the volumetric strain of the material.

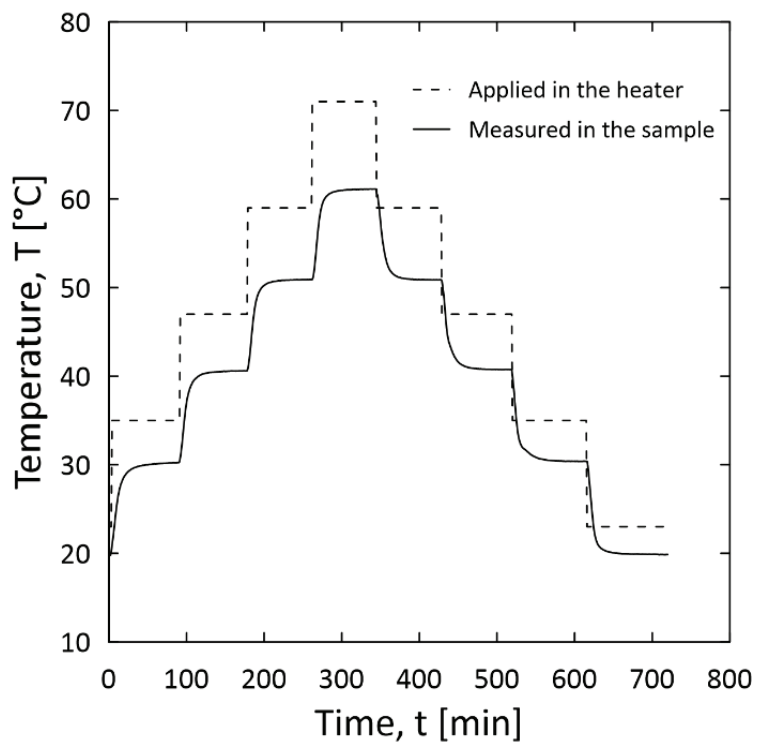

Fig. 5. The variations of the temperature measured in the trial specimen and the applied temperature in the heater for each thermal loading or unloading step

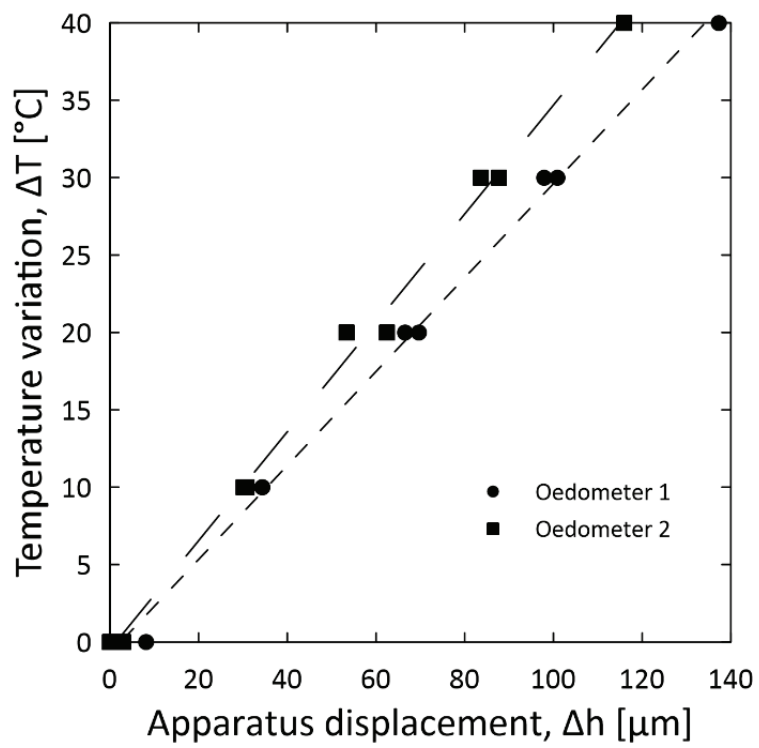

Fig. 6. Displacements induced by the thermal deformation of the oedometric apparatus

\section{Results and discussions}

In this section, the volume change behavior of saturated Foundry sand under non-isothermal conditions is investigated. In this context, the volume change behavior upon thermal loading is discussed first. Finally, the volume change behavior after thermal loading is expanded.

\subsection{Volume change behavior upon thermal loading}

The experimental variation of volumetric strain with temperature for the investigated coarse-grained soil is presented in Fig. 7. Under a nearly zero vertical stress, an expansive volume change occurs upon heating for all investigated relative densities. A contractive volume change occurs upon cooling.

The observation of an expansive volume change for loose coarse-grained soils upon heating is the first experimental account of this type available in the literature. All of the previous experimental investigations focusing on the volume change of loose coarse-grained soils (conducted at stress levels ranging from $50 \mathrm{kPa}$ up to $4 \mathrm{MPa}[3,4,6,7])$ reported a contractive volume change upon heating. This expansive behavior was only previously observed in loose granular columns of glass beads for stress levels lower than $10 \mathrm{kPa}$ [16].

Meanwhile, the observation of an expansive volume change for very dense coarse-grained soils upon heating was previously reported by $\mathrm{Ng}$ et al. [3] and Liu et al. [5]. As Sittidumrong et al. [4] showed that even very dense coarse-grained soils can contract upon heating under high stress levels (order of $\mathrm{MPa}$ ), it is hypothesized that the stress level, in conjunction with the relative density, may control the non-isothermal volume change behavior of coarse-grained soils subjected to monotonic heating, from contraction at high stress levels, towards expansion at low stress levels, for even very high relative densities.

Under the same value of vertical effective stress, the initial relative density appears to be the critical influencing factor for the magnitude of the expansion and contraction of coarse-grained soils. In this study, specimens of higher relative density exhibit more considerable expansion upon heating. For example, when the temperature variation reached $40{ }^{\circ} \mathrm{C}$, volumetric strains of $-0.201 \%,-0.219 \%$ and $-0.306 \%$ were measured for relative densities of $30 \%, 60 \%$ and $90 \%$, respectively.

\subsection{Volume change behavior after thermal loading}

As a smaller expansion upon heating is typically observed than the contraction upon cooling, an irreversible volume change is generated after one cycle of heating and cooling. This phenomenon is associated with irreversible residual contractive deformations after one heating-cooling cycle. This phenomenon was previously indicated in experimental tests on coarse-grained soils $[3,4]$ and other granular materials $[16,17]$, and is associated with particle rearrangement.

The preceding observations suggest that, under low values of vertical effective stress, the volume change behavior of very dense coarse-grained soils is nearly reversible upon heating and cooling, while the volume change behavior of loose coarse-grained soils is irreversible. It is hypothesized that there is a competing mechanism between thermal expansion of the material, resulting from thermal expansion of its constituents, and contractive reorganization of particles, induced by the alteration of the granular fabric and collapse of force 
chains. With this hypothesis, it appears that particle rearrangement is more important in loose specimens, which leads to a smaller expansion upon heating as compared to denser specimens, and a larger irreversible contraction after one heating and cooling cycle.

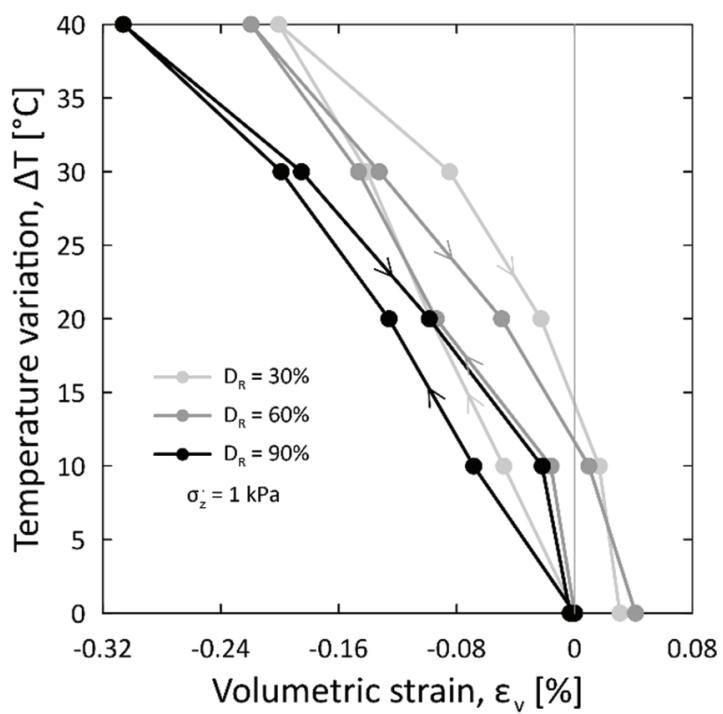

Fig. 7. Experimental response of Foundry sand subjected to heating and cooling at relative densities of $30 \%, 60 \%$, and $90 \%$

\section{Conclusions}

In this paper, the non-isothermal volume change behavior of saturated Foundry sand under low vertical effective stress is investigated using oedometric tests with temperature control. The main conclusions of this investigation can be summarized as follows:

(1) Heating coarse-grained soils under low values of vertical effective stress causes expansion. This phenomenon is observed for all of the considered values of relative density, which are associated with loose, medium dense and very dense states.

(2) Cooling coarse-grained soils under low values of vertical effective stress causes contraction. The magnitude of the contraction induced by cooling is typically slightly larger than the expansion caused by heating.

(3) The difference between the magnitudes of coolinginduced contraction and heating-induced expansion leads to an irreversible contractive volume change after one heating-cooling cycle.

(4) The initial relative density influences the volume change behavior of coarse-grained soils under nonisothermal conditions for the same (low) vertical effective stress: denser specimens exhibit more considerable thermal expansion than looser specimens and lower magnitude of irreversible contractive volume change after one heating-cooling cycle.
The authors are sincerely grateful to Prof. Lyesse Laloui, director of the Laboratory of Soils Mechanics at the Swiss Federal Institute of Technology in Lausanne, EPFL, for granting them access to the experimental facilities used to conduct the present work.

\section{References}

[1] L. Laloui and A. F. Rotta Loria, Analysis and Design of Energy Geostructures. (Elsevier Academic Press, 2019).

[2] A. F. Rotta Loria and J. B. Coulibaly, Geomech. Energy Environ. 10.1016/j.gete.2020.100193 (2020).

[3] C. W. W. Ng, S. H. Wang, and C. Zhou, Géotechnique Lett. 6, 124 (2016).

[4] J. Sittidumrong, A. Jotisankasa, and K. Chantawarangul, Geomech. Energy Environ. 100127 (2019).

[5] H. Liu, Y. Xiao, J. S. McCartney, and H. Liu, Geotech. Test. J. 41, (2018).

[6] K. M. Kosar, The Effect of Heated Foundations on Oil Sand, 1983.

[7] J. G. Agar, N. R. Morgenstern, and J. D. Scott, Can. Geotech. J. 23, 327 (1986).

[8] H. H. Vaziri and P. M. Byrne, Can. Geotech. J. 27, 802 (1990).

[9] Y. Pan, J. B. Coulibaly, and A. F. Rotta Loria, Géotechnique Lett. (2020).

[10] A. Di Donna and L. Laloui, Eng. Geol. 190, 65 (2015).

[11] Y. P. Vaid and D. Negussey, in Adv. Triaxial Test. Soil Rock (ASTM International, 1988).

[12] J. K. Mitchell and K. Soga, Fundamentals of Soil Behavior (John Wiley \& Sons Hoboken, NJ, 2005).

[13]ASTM, ASTM D4253-00 (2006).

[14]ASTM, ASTM D4254-06 (2006).

[15]C. Cekerevac, L. Laloui, and L. Vulliet, Geotech. Test. J. 28, 161 (2005).

[16] T. Divoux, H. Gayvallet, and J.-C. Géminard, Phys. Rev. Lett. 101, (2008).

[17] K. Chen, J. Cole, C. Conger, J. Draskovic, M. Lohr, K. Klein, T. Scheidemantel, and P. Schiffer, Nature 442, 257 (2006). 